\title{
疏水缔合聚丙烯酰胺与双子表面活性剂的相互作用
}

\author{
陈 洪 ${ }^{1, *}$ 李二晓 ${ }^{2}$ 叶仲斌 ${ }^{1,2}$ 韩利娟 $^{2}$ 罗平亚 ${ }^{1}$ \\ ('西南石油大学油气藏地质及开发工程国家重点实验室, 成都 610500; ${ }^{2}$ 西南石油大学化学化工学院, 成都 610500)
}

\begin{abstract}
摘要: 制备了一种脂肪酸酯双磺酸盐型双子表面活性剂, 利用粘度法、界面张力法和原子力显微镜研究了疏 水缔合聚丙烯酰胺与双子表面活性剂在溶液中的相互作用. 实验结果表明: 疏水缔合聚丙烯酰胺在溶液中能够 通过自组装形成疏水微区并发展成网络结构, 疏水微区与表面活性剂在溶液中能形成混合胶束; 当一定量的表 面活性剂加入时, 对疏水缔合聚丙烯酰胺的自组装起促进作用, 而过多双子表面活性剂的加入又会对聚合物分 子的自组装起抑制作用, 从而显著影响疏水缔合聚丙烯酰胺的溶液性质, 随着表面活性剂浓度的增加, 聚合物 溶液粘度先增加、再降低; 同时, 疏水缔合聚丙烯酰胺对双子表面活性剂的界面性能也有较大影响, 聚合物的加 入使双子表面活性剂降低油/水界面张力的能力下降, 油/水界面张力达到平衡所需时间延长.
\end{abstract}

关键词：双子表面活性剂； 疏水缔合聚丙烯酰胺；界面张力； 粘度；原子力显微镜 中图分类号: 0647

\section{Interaction of Hydrophobically Associating Polyacrylamide with Gemini Surfactant}

\author{
CHEN Hong ${ }^{1, *} \quad$ LI Er-Xiao ${ }^{2} \quad$ YE Zhong-Bin ${ }^{1,2} \quad$ HAN Li-Juan ${ }^{2} \quad$ LUO Ping-Ya $^{1}$
}

('State Key Laboratory of Oil and Gas Reservoir Geology and Exploitation, Southwest Petroleum University, Chengdu 610500, P. R. China; ${ }^{2}$ School of Chemistry and Chemical Engineering, Southwest Petroleum University, Chengdu 610500, P. R. China)

\begin{abstract}
A fatty acid disulfonate anionic gemini surfactant was prepared and the structure of the surfactant was characterized by ${ }^{1} \mathrm{H}-\mathrm{NMR}$. Hydrophobically associating polyacrylamide was prepared according to a procedure from literature. The interaction between the hydrophobically associating polyacrylamide (HAPAM) and the anionic gemini surfactant was studied by surface/interfacial tension, apparent viscosity, and atomic force microscopy (AFM). Experimental results show that HAPAM can form a network structure in the aqueous solution by self assembly. Mixed micelles are formed by the interaction of gemini surfactant micelles and the hydrophobic microdomain of the hydrophobically associating polyacrylamide in aqueous solution, which plays a remarkable role in the surfactant and polymer assembly. Mixed micelles can enhance the inter- or intra-molecular association between the polymer molecules and the surfactant, which increases the strength of the network formed by the hydrophobically associating polyacrylamide. The apparent viscosity of the solution increased by the addition of the gemini surfactant. When the addition of the gemini surfactant exceeded a certain amount, the association between the hydrophobic groups of the polymer decreased and the network formed by hydrophobically associating interaction was weakened by the surfactant micelles, which led to a decrease in solution viscosity. The polymer also largely influenced the interfacial properties of the gemini surfactant, especially the dynamical interfacial tension. A high polymer concentration led to an increase in the time required to reach equilibrium.
\end{abstract}

Received: October 27, 2010; Revised: December 14, 2010; Published on Web: January 24, 2011.

"Corresponding author. Email: chenh@swpu.edu.cn; Tel: +86-28-83034791.

The project was supported by the Youth Fund of Sichuan Province, China (08ZQ026-001).

四川省青年基金(08ZQ026-001)资助项目

(C) Editorial office of Acta Physico-Chimica Sinica 
Key Words: Gemini surfactant; Hydrophobically associating polyacrylamide; Interfacial tension; Viscosity; Atomic force microscopy

\section{1 引言}

疏水缔合水溶性聚合物(HAWSP) 是在聚合物 的亲水主链上引入极少量疏水基团(小于 $2 \%(x$, 摩 尔分数))而形成的一种水溶性聚合物, 在石油开采、 涂料、油漆、选矿、化妆品和药品制剂等领域具有广 阔的应用前景. ${ }^{1-2}$ HAWSP 由于疏水效应, 其溶液性 质具有不同于一般水溶性聚合物的特殊流变性, 一 直受到科学工作者极大的关注. ${ }^{3}$ 疏水改性聚丙烯酰 胺所形成的疏水缔合聚丙烯酰胺 (HAPAM) 是 HAWSP 中研究较多的一种. 它一般是通过丙烯酰 胺与含有双键的疏水单体共聚, 也可以化学改性, 在 聚丙烯酰胺(PAM)的链段上引入适当的疏水基团而 得到的. 虽然 HAPAM 与 PAM 在分子结构上有很大 相似之处, 但 HAPAM 表现出不同于 PAM 的良好的 增粘能力, ${ }^{4-6}$ HAPAM 在溶液中能够自组装, 形成空 间网状结构, 增大聚合物的流体力学体积, 从而表现 出良好的增粘、耐温、抗盐及抗剪切等性能, 使其成 为一种具有良好应用前景的水溶性聚合物材料.

普通的表面活性剂分子由一条疏水的碳链和一 个亲水头基组成不对称的“两亲” 结构, 而双子表面 活性剂 ${ }^{7-10}$ 的结构则较为特殊, 其分子中一般含有两 个亲水头基和两个疏水链, 在其亲水基或靠近亲水 基处, 由联结基团(spacer)通过共价键联结在一起. 双子表面活性剂由于结构独特, 比普通表面活性剂 具有更高的表/界面活性、更低的临界胶束浓度 (cmc)及具有许多特殊的性能. 就目前的报道来看, 阳离子型的双子表面活性剂的研究最多, 阴离子型、 非离子型及两性离子型的研究较少.

通常在水溶性聚合物中加表面活性剂, 使用这 种复配体系可以减少大分子或者表面活性剂的用 量, 改善溶液的性能, 显著提高体系的功效. ${ }^{.113}$ 并且 由于两者之间存在疏水或者静电相互作用, 还可能 使体系具有许多独特的性质, 例如体系的粘度、界面 吸附、增溶和药物传输等性能都不同于单一组分体 系, 因此表面活性剂/大分子混合体系的研究一直是 人们非常感兴趣的研究课题..$^{14-17}$ 国内外学者研究发 现疏水缔合聚合物与表面活性剂通过缔合作用会形 成混合胶束状聚集体, 随表面活性剂浓度的改变, 聚 合物/表面活性剂混合体系的溶液粘度会出现规律 性变化. ${ }^{18-20}$ 由于双子表面活性剂的特殊性能, 因此
研究双子表面活性剂与水溶性聚合物的相互作用显 得非常有实用价值. 本文制备了一种脂肪酸酯双磺 酸盐型双子表面活性剂, 研究了双子表面活性剂与 疏水缔合聚合物之间的相互作用, 以期为双子表面 活性剂/大分子混合体系在食品、日用化工以及油田 开发等领域的应用提供借鉴.

\section{2 实验部分}

\section{1 样品的合成及表征}

\subsection{1 疏水缔合聚丙烯酰胺的合成及表征}

投料比按表 1 , 参照文献 ${ }^{21}$ 给出的合成及提纯的 方法, 合成了疏水缔合聚丙烯酰胺(HAPAM)样品, 结构通式如图 1 所示.

参照 GB12005.1-89 规定的方法, 测得了疏水缔 合聚丙烯酰胺样品的特性粘数 $[\eta]$, 实验结果见表 1 .

\subsection{2 双子表面活性剂的合成及表征}

双十四酸乙二酯双磺酸盐型表面活性剂 (DMES-14)的合成方法参照专利. ${ }^{22}$ 对合成样品通过 核磁共振进行表征. ${ }^{1} \mathrm{H}$ NMR $\left(\mathrm{CDCl}_{3}, 300 \mathrm{~Hz}\right) \delta$ : 0.760 (s, 3H, CH H $_{3}, 1.180$ (m, 18H, $\mathrm{CH}_{3} \mathrm{CH}_{2}\left(\mathrm{CH}_{2}\right)_{9}-$ ), 1.36-1.46 (m, 2H, $\left.\mathrm{CH}_{3} \mathrm{CH}_{2}-\right), 2.26\left(\mathrm{~m}, 2 \mathrm{H},-\mathrm{CH}_{2}\right.$ $\left.\mathrm{CHSO}_{3} \mathrm{Na}\right), 3.42-3.56\left(\mathrm{~m}, 1 \mathrm{H},-\mathrm{CH}_{2} \mathrm{CHSO}_{3} \mathrm{Na}\right), 4.69$ (s, $2 \mathrm{H},-\mathrm{COOCH}_{2}-$ ). 确定了所合成样品的分子结 构式如图 2 所示.

\section{2 实验仪器及方法}

\section{2 .1 实验仪器和药品}

表 1 疏水缔合聚丙烯酰胺(HAPAM)的物性参数 Table 1 Characteristic parameters of hydrophobically associating polyacrylamide (HAPAM)

\begin{tabular}{ccccc}
\hline Sample & $w(\mathrm{AM}) / \%$ & $w(\mathrm{NaAA}) / \%$ & $w\left(\mathrm{C}_{16} \mathrm{DMAAC}\right) / \%$ & {$[\eta] /\left(\mathrm{mL} \cdot \mathrm{g}^{-1}\right)$} \\
\hline HAPAM & 75.0 & 24.9 & 0.1 & 964.3 \\
\hline
\end{tabular}

AM: acrylamide, NaAA: sodium acrylate,

$\mathrm{C}_{16}$ DMAAC: hydrophobic monomer; $[\eta]$ is intrinsic viscosity.

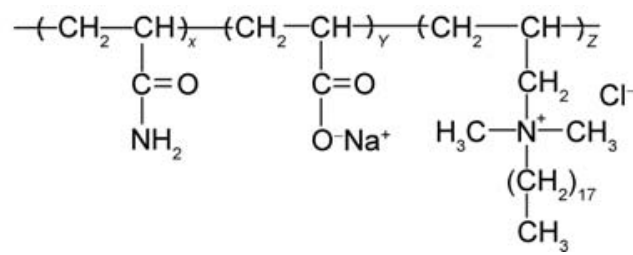

图 1 疏水缔合聚丙烯酰胺(HAPAM)分子结构式

Fig.1 Molecular structure of hydrophobically associating polyacrylamide (HAPAM) 


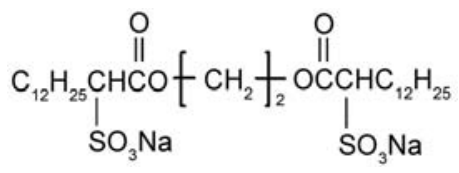

图 2 双子表面活性剂 DMES-14 分子结构式

Fig.2 Molecular structure of gemini surfactant DMES-14

美国 TX-500C 型旋转滴界面张力仪, 美国 Brookfield DV-III+型粘度计, 美国 Digital Instruments 公司原子力显微镜.

MES-14、正十二烷、丙烯酰胺(AM)和丙烯酸钠 (NaAA)均由成都科龙试剂公司提供, 分析纯.

2.2.2 表面张力和界面张力测定

用旋转滴法测定了双子表面活性剂 DMES-14 的表面张力和界面张力, 为了对比研究, 同时测定了 与阴离子双子表面活性剂结构相类似的普通表面活 性剂十四酸甲酯磺酸钠(MES-14)的表面张力和界 面张力. 实验中, 水相为蒸馏水, 油相为正十二烷, 测 试温度为 $(25.0 \pm 0.1)^{\circ} \mathrm{C}$.

\subsection{3 粘度测定}

使用 Brookfield 粘度计在 $25^{\circ} \mathrm{C}$ 、恒定转速(剪切 速率为 $7.34 \mathrm{~s}^{-1}$ )下测定不同表面活性剂浓度时表面 活性剂/大分子混合体系的表观粘度. 测试过程中保 持温度为 $(25.0 \pm 0.1){ }^{\circ} \mathrm{C}$.

2.2.4 表面活性剂/聚合物混合体系的溶液分子聚 集体形态

表面活性剂/聚合物混合体系的溶液分子聚集 体形态 ${ }^{23,24}$ 用原子力显微镜(AFM)观察, AFM 型号为 NanoscopeIIIa, 操作模式为轻敲模式. 轻敲模式既不 损坏样品表面, 又有较高的分辨率, 从而可用于表面 活性剂/聚合物混合体系的溶液分子聚集体形态研 究. ${ }^{25}$ 所用的针尖为氮化硅针尖, 力常数为 $0.106 \mathrm{~N}$. $\mathrm{m}^{-1}$, 针尖的曲率半径为 $27 \mathrm{~nm}$.

\section{3 结果及讨论}

\section{1 聚合物 HAPAM 对表面活性剂溶液性能的影响}

\section{1 .1 气/液界面性能}

用蒸馏水配制表面活性剂溶液, 在 $25^{\circ} \mathrm{C}$ 下, 用 TX-500C 型旋转滴界面张力仪测定溶液的表面张 力, 结果如图 3 所示.

从图 3 可以看出, 与疏水基相同的普通表面活 性剂相比, 双子表面活性剂DMES-14和普通表面活 性剂 MES-14 的临界胶束浓度 $(\mathrm{cmc})$ 时的界面张力 $\left(\gamma_{\mathrm{cmc}}\right)$ 差别不大, 但临界胶束浓度 $\mathrm{cmc}$ 却相差很大, 其 中, DMES-14 的临界胶束浓度为 $0.029 \mathrm{mmol} \cdot \mathrm{L}^{-1}$, 而

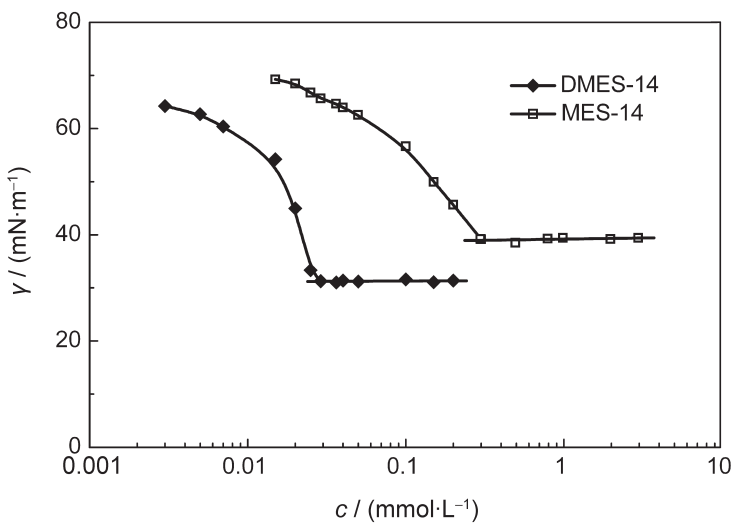

图3 界面张力 $(\gamma)$ 随表面活性剂溶液的浓度 $(c)$ 变化 Fig.3 Interfacial tensions $(\gamma)$ of surfactant solution as a function of concentration (c)

MES-14 的临界胶束浓度为 $0.31 \mathrm{mmol} \cdot \mathrm{L}^{-1}$, 两者相 差一个数量级, 说明双子表面活性剂 DMES-14 比普 通表面活性剂更容易在界面上吸附, 具有更高的降 低表面张力的能力.

\section{1 .2 液/液界面性能}

用蒸馏水配制表面活性剂 DMES-14 和聚合物 HAPAM 溶液, 以正十二烷为油相, 用 TX-500C 型旋 转滴界面张力仪测定溶液体系的油水界面张力, 实 验温度为 $25^{\circ} \mathrm{C}$, 结果如图 4、5 所示.

从图 4 可以看出, 油/水界面张力随着表面活性 剂浓度的增加而下降, 经过一个转折点之后, 界面 张力趋于平衡. 无聚合物时, 转折点对应的表面活 性剂浓度值最小, 随着聚合物浓度的增加, 其对应 的表面活性剂浓度值呈增加趋势, 表明缔合聚合物 HAPAM 在一定程度上影响了表面活性剂降低油水

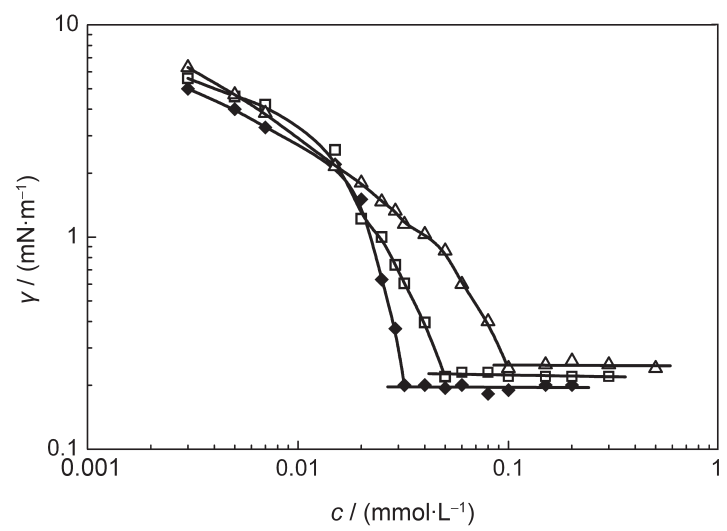

图 4 聚合物 HAPAM 对表面活性剂 DMES-14 溶液界面 张力的影响

Fig.4 Effect of HAPAM on interfacial tensions of DMES-14 solution

oil phase: dodecane; aqueous phase: $\bullet$ DMES-14, $\square$ DMES-14+ $500 \mathrm{mg} \cdot \mathrm{L}^{-1} \mathrm{HAPAM}, \triangle \mathrm{DMES}-14+1000 \mathrm{mg} \cdot \mathrm{L}^{-1} \mathrm{HAPAM}$ 
界面张力的能力. 出现这一现象的原因是由于缔合 聚合物 HAPAM 中含有疏水基团, 在体相中, 这些疏 水基团将与表面活性剂的疏水部分相互作用, 束缚 部分表面活性剂, 最终导致表面活性剂降低油水界 面张力的能力下降. 聚合物浓度越高, 这种束缚作用 越强, 需要 “消耗”更多的表面活性剂才能使油水界 面布满表面活性剂分子, 从而使油水界面张力降到 较低的值.

图 5 为聚合物 HAPAM 浓度对聚合物/表面活性 剂二元体系油水动态界面张力的影响的实验结果. 动态界面张力是指界面张力随时间的变化, 动态界 面张力特性可以反映表面活性剂降低界面张力到达 稳态值所需的时间, 反映表面活性剂体系在油水界 面的吸附特性以及吸附量的变化, 这对表面活性剂 的研究与应用同样具有重要意义. 因此, 有必要考察 缔合聚合物 HAPAM对表面活性剂降低油水界面张 力的动态特性的影响.

从图 5 可以看出, 当表面活性剂浓度一定时, 聚 合物浓度越低, 界面张力动态变化越快, 达到平衡所 需时间越少, 聚合物溶液的浓度越高, 界面张力下降 的速度越慢, 油/水界面张力达到平衡值所需时间越 长, 且界面张力最低值稍有升高. 其原因在于: 表面 活性剂与缔合聚合物存在较强的相互作用, 能够显 著地影响表面活性剂向油水界面扩散的速度, 此外, 聚合物的加入会使体系的粘度有一定程度的增加, 也会影响表面活性剂的扩散速度. 聚合物溶液浓度

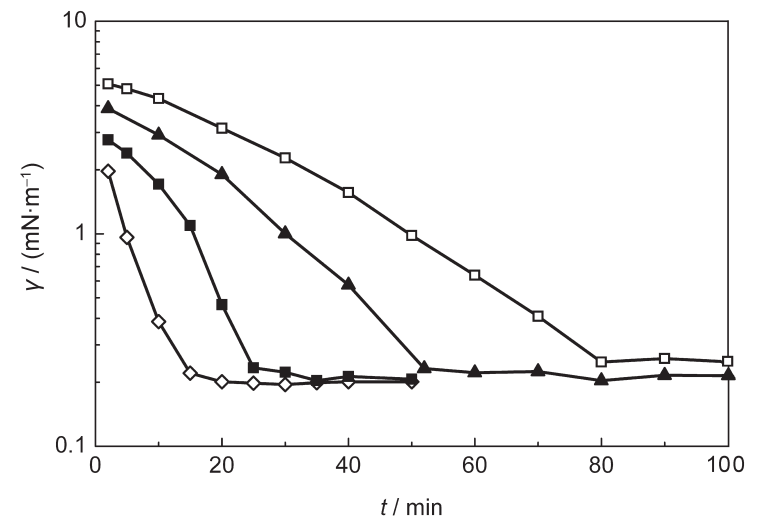

图 5 聚合物 HAPAM 浓度对聚合物/表面活性剂二元体系 油水动态界面张力的影响

Fig.5 Effect of HAPAM concentration on the dynamic interfacial tensions of polymer/surfactant binary system oil phase: dodecane; aqueous phase: $\diamond 0.29 \mathrm{mmol} \cdot \mathrm{L}^{-1} \mathrm{DMES}-14$,

- $0.29 \mathrm{mmol} \cdot \mathrm{L}^{-1} \mathrm{DMES}-14+100 \mathrm{mg} \cdot \mathrm{L}^{-1} \mathrm{HAPAM}$, $\Delta 0.29 \mathrm{mmol}$. $\mathrm{L}^{-1}$ DMES $-14+500 \mathrm{mg} \cdot \mathrm{L}^{-1} \mathrm{HAPAM}, \square 0.29 \mathrm{mmol} \cdot \mathrm{L}^{-1} \mathrm{DMES}-14+$ $1000 \mathrm{mg} \cdot \mathrm{L}^{-1} \mathrm{HAPAM}$
越高, 这种影响越大. 结果造成, 聚合物溶液浓度越 高, 达到平衡界面张力所需的时间越长.

\section{2 表面活性剂对聚合物溶液性能的影响}

HAPAM 分子链上同时带有亲水基团和疏水基 团, 由于疏水基团含量很低, 聚合物能够溶解在水 中. 疏水基团虽然被亲水链及其与极性环境的亲和 力拉到水中, 但疏水基团仍具有逃逸极性环境的趋 势. 疏水基团为了在水溶液中保持稳定, 要求尽量减 少与水的接触面积, 疏水基团会在水分子的“驱动” 或“疏水效应”下靠在一起, 疏水基团相互靠拢、缔合 形成“疏水微区”. 在表面活性剂和 HAPAM 的混合 体系中, 表面活性剂的疏水部分与聚合物的疏水基 团两者之间存在疏水相互作用, 在溶液中容易形成 混合胶束, 表面活性剂的存在将会影响缔合聚合物 的自组装行为, 从而对聚合物溶液的宏观性能产生 影响. 因此, 研究中考察了当聚合物溶液浓度为 $1000 \mathrm{mg} \cdot \mathrm{L}^{-1}$, 表面活性剂浓度变化对聚合物溶液性 能的影响, 表面活性剂浓度范围为 0.002-2 mmol $\mathrm{L}^{-1}$, 测试温度 $25^{\circ} \mathrm{C}$, 实验结果如图 6 所示.

从图 6 可以看出, 随着表面活性剂浓度的增加, 聚合物溶液粘度出现先增加、然后再降低的过程. 体 系粘度的最大值对应的表面活性剂浓度为 0.05 $\mathrm{mmol} \cdot \mathrm{L}^{-1}$, 大约是表面活性剂 $\mathrm{cmc}$ 的 1.7 倍. 其原因 在于, 由于 HAPAM 为疏水缔合聚合物, 在溶液中能 够通过自身的缔合作用而聚集形成“疏水微区”. 如 果缔合作用发生在聚合物的自身链内, 会导致聚合 物分子卷曲, 溶液粘度下降, 若缔合作用发生在聚合 物分子之间, 聚合物容易形成超分子结构, 增加溶液 粘度. 实验中聚合物溶液浓度为 $1000 \mathrm{mg} \cdot \mathrm{L}^{-1}$, 加入

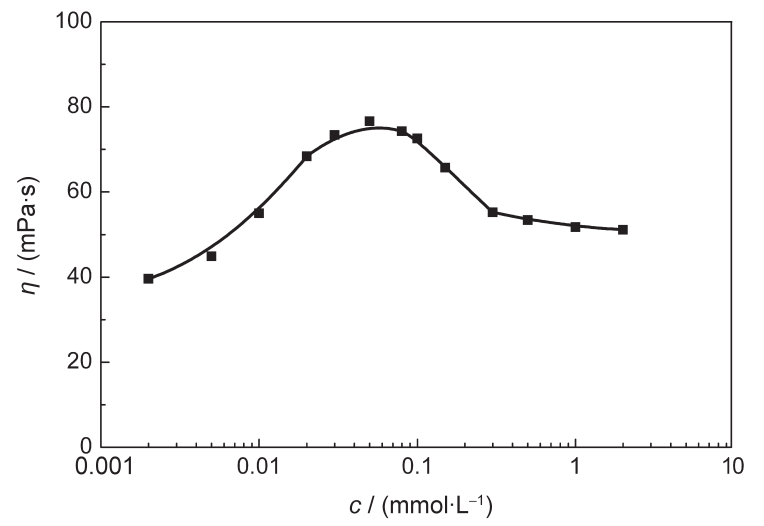

图 6 表面活性剂浓度对聚合物溶液粘度的影响

Fig.6 Effect of surfactant concentration on the viscosity of polymer solution $1000 \mathrm{mg} \cdot \mathrm{L}^{-1} \mathrm{HAPAM}$ 
(a)

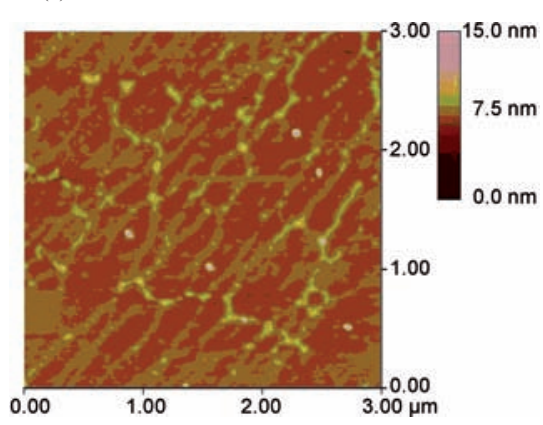

(b)

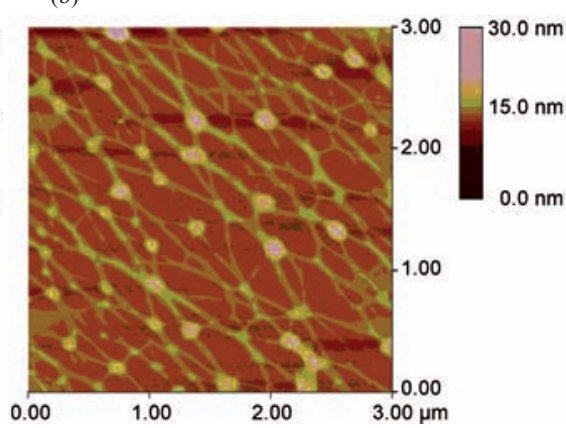

图 7 聚合物的 AFM图 (c)

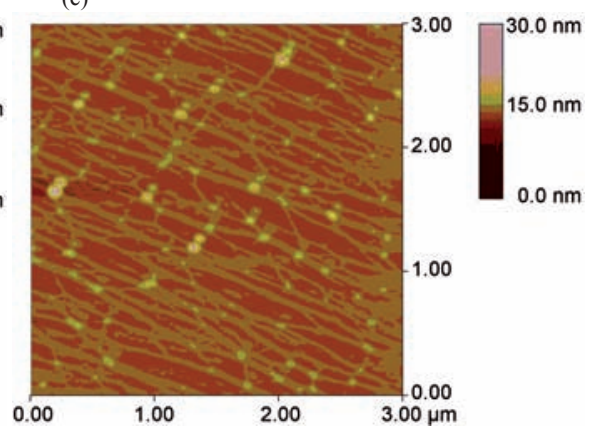

Fig.7 AFM images of polymers

(a) $1000 \mathrm{mg} \cdot \mathrm{L}^{-1} \mathrm{HAPAM}$, (b) $1000 \mathrm{mg} \cdot \mathrm{L}^{-1} \mathrm{HAPAM}+0.05 \mathrm{mmol} \cdot \mathrm{L}^{-1} \mathrm{DMES}-14$, (c) $1000 \mathrm{mg} \cdot \mathrm{L}^{-1} \mathrm{HAPAM}+0.3 \mathrm{mmol} \cdot \mathrm{L}^{-1} \mathrm{DMES}-14$

表面活性剂浓度较低时, 表面活性剂会与缔合聚合 物产生的 “疏水微区”相互作用形成混合胶束, 混合 胶束中高分子疏水链的密度大, 表面活性剂的加入 对缔合聚合物分子间的聚集起促进作用, 表现出随 表面活性剂浓度的增大溶液粘度增大; 当表面活性 剂浓度超过一定值后, 表面活性剂与聚合物链段上 的疏水基团相互作用进一步增强, 混合胶束中表面 活性剂的密度增大, 使混合胶束中的共聚物大分子 链上的部分疏水侧链单独地增溶于表而活性剂胶束 中, 从而对聚合物分子链之间的缔合相互作用产生 了屏蔽效应, 聚合物分子间作用减弱, 使部分高分子 聚集体开始解离, 交联的聚合物网络受到破坏与解 体, 导致溶液粘度下降; 随着表面活性剂浓度的继 续增大, 此阶段可能由于溶液中存在可解离的高分 子聚集体不断减少, 新增的表面活性剂所形成的聚 集体对溶液粘度的影响程度也逐渐降低.

\section{3 表面活性剂/聚合物混合体系的溶液分子聚集} 体形态

疏水缔合聚合物 HAPAM 与双子表面活性剂的 相互作用, 对聚合物和表面活性剂各自的性质均产 生了影响, 特别是表面活性剂对聚合物溶液的粘度 影响更为显著, 而聚合物溶液的粘度性质是其分子 聚集体形态的宏观表现, 因此, 利用原子力显微镜研 究了表面活性剂的加入对聚合物分子聚集体形态的 影响, 实验结果如图 7 所示.

图 7(a)为聚合物的 AFM 照片, 从图中可以看 出, 在纯水中, 缔合聚合物 HMPAM 已形成了明显的 空间网络结构. 图 7(b) 为在疏水缔合聚丙烯酰胺溶 液中加入 $0.05 \mathrm{mmol} \cdot \mathrm{L}^{-1}$ 表面活性剂的 $\mathrm{AFM}$ 照片, 此时表面活性剂浓度正好是图 6 中粘度最大值所对 应的表面活性剂浓度. 对比图 7(a)和 7(b) 可以看出,
由于双子表面活性剂的加入, 体系中空间网络结构 的网孔更加密集, 且连接网孔的链束较粗, 特别是链 束相交的部位, 因此, 宏观上表现出表面活性剂/聚 合物溶液混合体系的粘度变大. 图 7(c) 是疏水缔合 聚丙烯酰胺溶液中加入 $0.3 \mathrm{mmol} \cdot \mathrm{L}^{-1}$ 表面活性剂的 AFM 照片. 对比图 7(c) 和图 7(b), 可以看出, 随着表 面活性剂的继续加入, 体系中空间网络结构的网孔 变得较稀疏, 链束较细, 而宏观上也表现出表面活性 剂/聚合物溶液混合体系的粘度较小. 聚合物溶液的 AFM 图与聚合物溶液粘度实验的结果(图 6)相一 致. 实验结果表明: 疏水缔合聚合物在溶液中能够 通过自组装作用形成网络结构, 一定量表面活性剂 的加入, 会对这种自组装起促进作用, 而过多表面活 性剂的加入会对聚合物分子的自组装起抑制作用, 从而能很好地解释表面活性剂对缔合聚合物溶液粘 度影响的原因。

\section{4 结 论}

(1) 制备了一种脂肪酸酯双磺酸盐型双子表面 活性剂DMES-14. 与疏水基相同的普通表面活性剂 相比, 双子表面活性剂DMES-14和普通表面活性剂 MES-14 的 $\gamma_{\mathrm{cmc}}$ 差别不大, 但 DMES-14 的临界胶束浓 度比 MES-14 低一个数量级.

(2) 双子表面活性剂 DMES-14 可以将正十二 烷/水界面张力降到 $0.2 \mathrm{mN} \cdot \mathrm{m}^{-1}$, 说明 DMES-14 具 有良好的降低油水界面张力的能力.

(3) 聚合物 HAPAM 的加入对表面活性剂 DMES-14 界面活性有一定降低, 且聚合物浓度越 高, 达到平衡界面张力所需的时间越长.

(4) 当表面活性剂 DMES-14 浓度较低时, 随着 DMES-14 浓度的增加, 聚合物 HAPAM 溶液粘度增 
加; 当表面活性剂浓度为 $0.05 \mathrm{mmol} \cdot \mathrm{L}^{-1}$, 大约是表 面活性剂 $\mathrm{cmc}$ 的 1.7 倍时, 聚合物溶液粘度达到最 高; 当表面活性剂浓度继续增大, 聚合物溶液粘度 开始下降, 直至随表面活性剂浓度的增加聚合物溶 液粘度不再发生变化.

\section{References}

(1) Glass, J. E. Polymers in Aqueous Media, ACS Symp. Ser. Vol.233; American Chemical Society: Washington DC, 1989; p 103

(2) McCormick, C. L.; Block, J.; Schulz, D. N. Water-Soluble Polymers. In Encyclopedia of Polymer Science and Engineering; Mark, H. F., Bikales, N. M., Overberger, C. G., Menges, G. Eds.; Wiley-Interscience: New York, 1989; Vol.17, p 730

(3) Francoise, W. M.; Sudarshi, R. T. A.; Desmond, G. E. Langmuir 1997, 13, 111.

(4) Klucker, R.; Schosseler, F. Macromolecules 1997, 30, 4927.

(5) Nilsson, S.; Thuresson, K.; Lindman, B.; Nystrom, B. Macromolecules 2000, 33, 9641.

(6) Beaudoin, E.; Hiorns, R. C.; Borisov, O.; Francois, J. Langmuir 2003, 19, 2058 .

(7) Chen, H.; Han, L.; Luo, P.; Ye, Z. Surf. Sci. 2004, 552, L53.

(8) Zana, R. Adv. Colloid Interface Sci. 2002, 97, 205.

(9) Menger, F. M.; Keiper, J. S. Angew. Chem. Int. Edit. 2000, 39, 19060.

(10) Menter, F. M.; Mbadugha, B. N. A. J. Am. Chem. Soc. 2001, 123,875 .

(11) Deo, P.; Somasundaran, P. Langmuir 2005, 21, 3950.

(12) Nizri, G.; Lagerge, S.; Kamyshny, A.; Major, D. T.; Magdassi, S. J. Colloid Interface Sci. 2008, 320, 74.

(13) Goddard, E. D. J. Colloid Interface Sci. 2002, 256, 228.

(14) Wang, D. X.; Luo, L.; Zhang, L.; Wang, Y. Y.; Zhao, S.; Yu, J. Y. Acta Phys. -Chim. Sin. 2005, 21, 1205. [王东贤, 罗 澜, 张 路, 王宜阳, 赵 濉, 俞稼镛. 物理化学学报, 2005, 21,
1205.]

(15) Zhang, J.; Xie, X. M.; Li, Z. M.; Zhang, L. M.; Li, J.; Luo, P. Y. Acta Phys. -Chim. Sin. 2002, 18, 455. [张 健, 谢续明, 李卓 美, 张黎明, 李 健, 罗平亚. 物理化学学报, 2002, 18, 455.]

(16) Dai, Y. H.; Wu, F. P.; Li, M. Z.; Wang, E. J. Acta Chimica Sinica 2005, 63, 1329. [戴玉华, 吴飞鹏, 李妙贞, 王尔鑑. 化学学报, 2005, 63, 1329.]

(17) Jiang, L. D.; Gao, B. J.; Li, G. Acta Phys. -Chim. Sin. 2007, 23, 377. [江立鼎, 高保娇, 李 刚. 物理化学学报, 2007, 23, 377.]

(18) Smith, G. L.; McCormick, C. L. Langmuir 2001, 17, 1719.

(19) Sastry, N. V.; Hoffmann, H. Colloids and Surfaces A: Physicochemical and Engineering Aspects 2004, 250, 247.

(20) Jiménez-Regalado, E.; Selb, J.; Candau, F. Langmuir 2000, 16, 8611.

(21) Chen, H.; Han, L. J.; Xu, P.; Luo, P. Y. Acta Phys. -Chim. Sin. 2003, 19, 1020. [陈 洪, 韩利娟, 徐 鹏, 罗平亚. 物理化学 学报, 2003, 19, 1020.]

(22) Chen, H.; Ye, Z. B.; Fan, L.; Han, L. J. The Preparation of a Sulphonated Fatty Acid Double Ester Gemini Surfactant. CN Patent 101357307A, 2009-02-04. [陈 洪, 叶仲斌, 樊 力, 韩利娟. 一种脂肪酸双酯双磺酸盐表面活性剂及制备方法: 中国, CN 101357307A [P]. 2009-02-04.]

(23) Wang, Y.; Li, Y. S.; Song, R.; Shen, J. S.; Long, C. F. Chem. J. Chin. Univ. 2001, 22, 1940. [王 铀, 李英顺, 宋 锐, 沈静 姝, 龙程奋. 高等学校化学学报, 2001, 22, 1940.]

(24) Xu, P. Study on the Microstructure of Hydrophobically Associating Water-Soluble Polymer Solution and the Influence of Surfactant on Its Rheology. Ph.D. Dissertation, Southwest Petroleum University, Chengdu, 2001. [徐 鹏. 疏水缔合水 溶性聚合物溶液微观结构研究及表面活性剂对其流变性的影 响[D]. 成都: 西南石油大学, 2001.]

(25) Xin, X. The Physicochemical Properties of Water-Soluble Polymer and Its Interaction with Carboxylate Surfactant. Ph.D. Dissertation, Shangdong University, Jinan, 2008. [辛 霞. 水 溶性高分子的物化性质及其与羧酸盐表面活性剂的相互作用 [D]. 济南: 山东大学, 2008.] 